\title{
Fatores de risco para metástases à distância no câncer de cabeça e pescoço
}

\author{
Risk factors for distant metastasis in head and neck cancer
}

Rogério Aparecido Dedivitis; Odilon Victor Porto Denardin²; Mário Augusto Ferrari de Castro33; Elio Gilberto Pfuetzenreiter JR4

RES U M O

\begin{abstract}
Objetivo: Identificar os fatores de risco para o desenvolvimento de metástase à distância (MD) entre pacientes com câncer de cabeça e pescoço cirurgicamente tratados. Métodos: Realizou-se estudo retrospectivo de 253 pacientes entre janeiro de 1997 e dezembro de 2002. Deles, 10 desenvolveram MD durante o seguimento. Foram avaliados: gênero, idade, sítio primário, classificação TNM, nível de doença linfonodal e presença de invasão extracapsular macro ou microscópica. Resultados: A média de sobrevida para pacientes com MD, a partir da finalização do tratamento oncológico, foi de 16 meses (com desvio-padrão de 2,256 meses). A presença de invasão extracapsular macro (risco relativo $=14,429)$ e microscópica $(R R=17,471$ ) e os estadiamentos patológicos avançados cervical $(R R=4,409)$ e do tumor primário ( $R R=2,929)$ apresentaram significado estatístico como fatores de risco para MD. Conclusão: Os fatores de risco para o surgimento de MD foram: invasão extracapsular macro e microscópica, presença de linfonodo positivo à avaliação histopatológica e tumor primário avançado.
\end{abstract}

Descritores: Neoplasias de Cabeça e Pescoço. Metástase Neoplásica. Estadiamento de Neoplasias.

\section{INTRODUÇÃO}

A incidência de metástases à distância (MD) tem aumentado com o progressivo desenvolvimento do controle loco-regional do carcinoma espinocelular (CEC) de cabeça e pescoço como consequência da multimodalidade de tratamentos. Como a perspectiva de cura é remota dentre os pacientes com MD, o reconhecimento dos fatores de risco para o desenvolvimento dessa situação é necessário para estratificar os pacientes que poderiam beneficiar-se de terapia sistêmica ${ }^{1}$.

O objetivo desse trabalho é identificar os fatores de risco para o desenvolvimento de MD entre os pacientes com CEC de cabeça e pescoço cirurgicamente tratados.

\section{MÉTODOS}

Realizou-se estudo retrospectivo de uma coorte de 253 pacientes sem tratamento prévio, portadores de carcinoma espinocelular de vias aerodigestivas superiores, submetidos ao tratamento cirúrgico isolado ou combinado com radioterapia adjuvante, entre janeiro de 1997 e dezembro de 2002, nos serviços de Cirurgia de Cabeça e Pescoço do Hospital Ana Costa, e da Irmandade da Santa
Casa da Misericórdia de Santos. Deles, 10 desenvolveram, ao seguimento, MD.

Pacientes que apresentaram MD foram comparados com aqueles que não apresentaram, com respeito aos seguintes aspectos clínicos e histopatológicos: gênero, idade, sítio primário, classificação TNM, nível de doença linfonodal e presença de invasão extracapsular macro ou microscópica.

Conforme nossa rotina de seguimento, pacientes tratados por câncer das vias aerodigestivas superiores são avaliados mensalmente no primeiro ano, bimestralmente no segundo ano, trimestralmente daí até completar o quinto anos após o tratamento e, a partir de então, as visitas são anuais. Os pacientes são submetidos a exame loco-regional completo, incluindo exame videoendoscópico da mucosa das vias aerodigestivas superiores a cada vez. Ultra-sonografia com Doppler colorido da região cervical é feita a cada duas consultas. Radiografias simples de tórax é feita a cada seis meses, enquanto a tomografia computadorizada da região de cabeça e pescoço, anualmente. Outros métodos de investigação são seletivamente indicados.

O tempo de seguimento considerado foi o intervalo entre a data de finalização do tratamento oncológico com finalidade de cura e a data do óbito ou última informação quando sob censura. A estatística descritiva foi con-

\footnotetext{
Trabalho realizado no Curso de Pós-graduação em Ciências da Saúde do Hospital Heliópolis HOSPHEL, São Paulo; Serviços de Cirurgia de Cabeça e Pescoço do Hospital Ana Costa e da Irmandade da Santa Casa da Misericórdia de Santos, Santos - SP - BR.

1. Livre Docente pela Faculdade de Ciências Médicas da Fundação Lusíadas - UNILUS, Santos - SP - BR. 2. Doutor em Medicina pelo Curso de Pósgraduação em Endocrinologia da Universidade Federal de São Paulo UNIFESP - São Paulo - SP - BR. 3. Residente de Cirurgia de Cabeça e Pescoço do Hospital Ana Costa, Santos - SP - BR. 4. Mestre pelo Curso de Pós-Graduação em Ciências da Saúde do Hospital Heliópolis - HOSPHEL - São Paulo - SP - BR.
} 
templada pela distribuição de frequências para as variáveis categóricas e as medidas de tendência central e de variabilidade para as numéricas ou continuas. As probabilidades de sobrevida foram estimadas pelo método de Kaplan-Meier. Os fatores de risco foram avaliados pelo teste do qui-quadrado, sendo calculados os riscos relativos para o desenvolvimento de MD.

\section{RESULTADOS}

A distribuição das MD foi para: pulmões (cinco casos), sistema nervoso central (três), osso (um) e pele (um). A média de sobrevida para pacientes com MD, a partir da finalização do tratamento oncológico, foi de 16 meses (com desvio-padrão de 2,256 meses) (Figura 1).

A tabela 1 mostra a distribuição dos pacientes de acordo com os diversos fatores analisados e a ocorrência de MD e a tabela 2 apresenta sua distribuição quanto ao sítio primário. A presença de invasão extracapsular macro e microscópica e a localização do tumor primário (oculto e recesso piriforme) apresentaram significado estatístico como fatores de risco para MD. A tabela 3 apresenta os respectivos fatores de risco.

\section{DISCUSSÃO}

Na ausência de evidência clínica de doença loco-regional, quando ocorrem MD, estas estão supostamente presentes desde o início do tratamento. A prevalência de MD à necrópsia (de 37\% a 57\%) é muito maior do que a observada em estudos clínicos². A incidência global de MD varia de $5 \%$ a $14 \%$. Os sítios mais comuns são os pulmões ( $52 \%$ a $83 \%$ ) e ossos ${ }^{2-6}$. Encontramos, nesta série, um índice de 3,95\% de MD os sítios mais comumente acometidos foram os pulmões (50\%) e sistema nervoso central (30\%).

Os seguintes fatores, nesta série, foram considerados significativos como de risco para o surgimento de

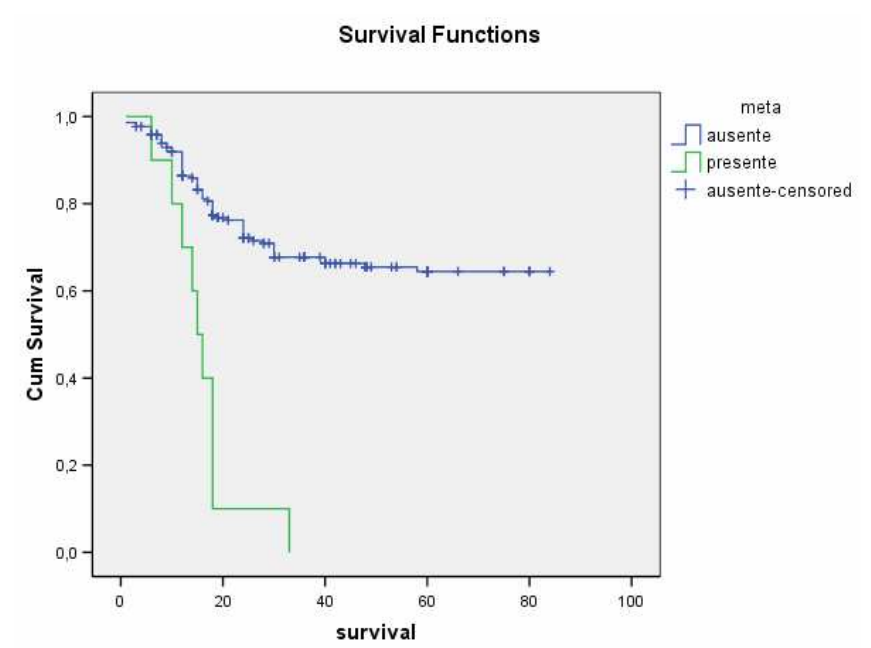

Figura 1 - Curva de sobrevida para os pacientes com e sem MD.
Tabela 1 - Variáveis estudadas como fatores de risco para o desenvolvimento de MD.

\begin{tabular}{|c|c|c|c|c|}
\hline \multirow[t]{2}{*}{ Variável } & & \multicolumn{2}{|c|}{ MD } & \multirow[t]{2}{*}{$p$} \\
\hline & & Ausente & Presente & \\
\hline \multirow[t]{2}{*}{ Gênero } & masculino & 182 & 10 & 0,365 \\
\hline & feminino & 33 & 0 & \\
\hline \multirow[t]{2}{*}{ Idade } & $<55$ & 55 & 3 & 0,719 \\
\hline & $>55$ & 161 & 7 & \\
\hline \multicolumn{5}{|l|}{ Estadiamento $\mathrm{T}$} \\
\hline \multirow[t]{4}{*}{ (patológico) } & $\mathrm{T} 1$ & 61 & 0 & 0,431 \\
\hline & $\mathrm{T} 2$ & 62 & 2 & \\
\hline & T3 & 64 & 3 & \\
\hline & $\mathrm{T} 4$ & 20 & 1 & \\
\hline \multicolumn{5}{|l|}{ Estadiamento $\mathrm{N}$} \\
\hline \multirow[t]{4}{*}{ (patológico) } & No & 150 & 4 & 0,280 \\
\hline & N1 & 10 & 0 & \\
\hline & N2 & 49 & 5 & \\
\hline & N3 & 7 & 1 & \\
\hline \multirow[t]{2}{*}{ IEC macroscópica } & ausente & 202 & 5 & $<0,001$ \\
\hline & presente & 14 & 5 & \\
\hline IEC microscópica & ausente & 198 & 4 & $<0,001$ \\
\hline
\end{tabular}

Tabela 2 - Sítio primário como fator de risco para o desenvolvimento de MD.

\begin{tabular}{ccc}
\hline \multicolumn{2}{c}{$M D$} & $p$ \\
\cline { 2 - 3 } & ausente presente \\
\hline
\end{tabular}

\begin{tabular}{lrll}
\hline$>55$ & 161 & 7 & 0,004 \\
lábio inferior & 5 & 0 & \\
língua & 23 & 0 & \\
soalho de boca & 12 & 0 & \\
região jugal & 1 & 0 & \\
palato duro & 2 & 0 & \\
loja amigdalina & 28 & 0 & \\
palato mole & 4 & 0 & 1 \\
base de língua & 7 & 1 \\
glote & 84 & 2 \\
supraglote & 28 & 2 \\
recesso piriforme & 4 & 0 \\
seios paranasais & 5 & 4 \\
primário oculto & 9 & 0 \\
infraglote & 4 &
\end{tabular}

Tabela 3 - Risco relativo para o desenvolvimento de MD.

\begin{tabular}{lc}
\hline Fator & Risco relativo \\
\hline pT avançado (T3 / T4) & 2,929 \\
pN + & 4,409 \\
IEC grosseira & 14,429 \\
IEC microscópica & 17,471 \\
\hline
\end{tabular}


MD: presença de invasão extracapsular (tanto macro como microscópica) e estadiamento loco-regional patológico avançado - tumor primário T3 e T4 e presença de metástases cervicais.

Em uma análise multivariada, os mais significativos fatores de risco para MD foram o número de níveis cervicais envolvidos e o sítio primário do tumor (carcinoma de hipofaringe, risco relativo $=2,8$ ). Pacientes com mais de um nível linfonodal com metástase apresentaram risco relativo de 3,177. Em outra série, de 114 pacientes previamente não tratados e sob estádios clínicos III e IV, com tumores de cavidade oral, orofaringe, hipofaringe e laringe, submetidos à multimodalidade de tratamento, 20 desenvolveram MD. A incidência de MD foi maior entre os pacientes que apresentaram linfonodos cervicais clinicamente positivos em comparação aos pacientes estadiados como tendo pescoço negativo clinicamente (25\% vs. $4 \%$, p < $0,05)$ e especialmente naqueles pacientes que apresentaram múltiplos níveis cervicais com metástases quando comparado com aqueles sem metástase ou com um único nível positivo $(35 \% \text { vs. } 5 \%, p<0,05)^{8}$. A hipofaringe e a supraglote foram consideradas os sítios primários com maior propensão a desenvolver MD em outra série ${ }^{2}$. Para outros autores, o principal fator preditivo para MD foi o volume tumoral $(p<0,0001)^{9}$.

Na análise de 873 pacientes tratados com radioterapia definitiva e com seguimento superior a dois anos, foram avaliados os fatores de risco que poderiam influenciar o surgimento de MD. A análise multivariada mostrou que gênero $(p=0,0390)$, estádio $T(p<0,0001)$, estádio $N$ $(p=0,0060)$, nível linfonodal $(p<0,0001)$ e controle locoregional $(p<0,0001)$ significantemente influenciaram para o paciente estar livre de MD. A análise multivariada mostrou que gênero $(p=0,0049)$, estádio $T(p<0, .0001)$, estádio $N(p<0,0001)$ e controle loco-regional ( $p<0,0001)$ influenciaram significativamente a sobrevida causa-específica $^{10}$

Tumores da cabeça e do pescoço estão sob risco de desenvolver MD antes, durante ou após o tratamento oncológico. Nessa situação, os pacientes têm prognóstico reservado, com uma sobrevida média de 4,3 a 7,3 meses e o tratamento é habitualmente paliativo ${ }^{11}$. 0 tempo médio de ocorrência de MD em nossa casuística foi de 16 meses e o tempo médio de sobrevida, uma vez estabelecido o diagnóstico, foi de cinco meses. Estratégias terapêuticas que objetivem a diminuição da incidência de MD em pacientes com carcinomas avançados de cabeça e pescoço devem ser consideradas em pacientes de alto risco.

Não existe uma abordagem padronizada e baseada em evidências para rastreamento das $\mathrm{MD}^{12}$. Tal estratégia permitiria um aconselhamento aos pacientes acerca das decisões terapêuticas e teria impacto econômico nos cuidados à saúde. O tipo histológico e o estádio da doença são críticos, pois se correlacionam ao risco de $\mathrm{MD}$. O sítio mais comumente acometido são os pulmões e o método validado para sua detecção é a tomografia computadorizada. Avanços recentes, como a tomografia por emissão de pósitrons podem tornar-se rotina diagnóstica. As recomendações para rastreamento de rotina seguem subjetivas e história clínica e exame físico cuidadosos seguem sendo a base do seguimento, já que a sensibilidade e a relação de custo e benefício dos estudos de imagem permanecem controversos $^{13}$. Em análise de 109 pacientes consecutivos portadores de câncer de cabeça e pescoço, o estudo tomográfico revelou metástases pulmonares em 20 (18) e hepática em um paciente. Outros nove (11\%) tiveram investigação tomográfica negativa antes do tratamento cirúrgico, porém, desenvolveram MD durante os 12 meses seguintes. Assim, a sensibilidade da tomografia computadorizada foi de $73 \%$ e a especificidade de $80 \%$ na detecção de MD $^{14}$. Em 92 pacientes consecutivos estudados com câncer de cabeça e pescoço e com fatores de risco para o desenvolvimento de $\mathrm{MD}$, foi realizado o rastreamento de corpo inteiro com FDG-PET, com identificação pré-tratamento em 19 deles (21\%), com resultados superiores aos obtidos pela tomografia computadorizada quanto à sensibilidade (53\% vs. $37 \%$ ) e valor preditivo para o teste positivo ( $80 \%$ vs. $75 \%$ ), no entanto, a combinação dos dois métodos resultou em sensibilidade de $63 \%{ }^{15}$.

A ressecção de metástase pulmonar em paciente com CEC primário oferece um aumento de sobrevida quando comparada com os esquemas de quimioterapia atuais, estando indicada naqueles com condições clínicas adequadas e que não apresentem doença extra-pulmonar ${ }^{16}$. O tratamento adjuvante visa, teoricamente, controlar manifestação sistêmica da doença, ou seja, metástase, que em geral não é detectada. Nesse sentido, o efeito da quimioterapia adjuvante dificilmente é mensurado e seu uso acrescenta ainda toxicidade ao paciente. Até o momento, a presença de MD traduz-se para esses pacientes em uma expectativa de vida medida em meses, sendo o tratamento puramente paliativo. A metástase pulmonar isolada pode ser removida com sucesso, resultando em longo controle da doença em pacientes selecionados. Radioterapia pode ser empregada para paliação de metástases ósseas e, ocasionalmente, para metástases pulmonares e cerebrais. A quimioterapia não tem maior impacto atualmente, com exceção dos casos cujo tumor primário é de rinofaringe. Os cuidados sintomáticos paliativos, com controle adequado da dor, são essenciais ${ }^{17}$.

Os fatores de risco para o surgimento de MD em pacientes tratados cirurgicamente por carcinoma espinocelular das vias aerodigestivas superiores foram: invasão extracapsular microscópica (risco relativo de 17,471) e macroscópica (RR de 14,429), presença de linfonodo positivo à avaliação histopatológica (RR de 4,409) e tumor primário avançado, ou seja T3 e T4 (RR de 2,929). 


\section{A B S T R A C T}

Objective: To identify risk factors for the development of distant metastasis (MD) among patients with head and neck squamous cell carcinoma (SCC) surgically treated. Methods: A retrospective study of 253 patients from January, 1997 to December, 2002 was performed. Out of them, 10 patients presented DM during the follow up. The following aspects were analyzed: gender, age, tumor primary site, TNM classification, cervical lymph node status, and the presence of extracapsular spread - gross or microscopic. Results: The survival average for the patients with DM, after finishing the oncological treatment, was 16 months (standard deviation $=2.256$ ). The presence of both gross extracapsular spread (Odd ratio $=14.429$ ) and the microscopic one $(O R=17.471)$ and the both the neck advanced pathological staging $(O R=4.409)$ and the primary tumor staging $(R R=2.929)$ presented statistical significance as risk fators for the occurrence of DM. Conclusion: The risk factors for DM were: microscopic and gross extracapsular spread, the pathological presence of positive neck lymph node and advanced primay tumor.

Key words: Head and Neck Neoplasms. Neoplasm Metastasis. Neoplasm Staging.

\section{REFERENCIAS}

1. Alvi A, Johnson JT. Development of distant metastasis after treatment of advanced-stage head and neck cancer. Head Neck. 1997;19(6):500-5.

2. León X, Quer M, Orús C, del Prado Venegas M, López M. Distant metastases in head and neck cancer patients who achieved locoregional control. Head Neck. 2000;22(7):680-6.

3. Jones AS, Roland NJ, Field JK, Phillips DE. The level of cervical lymph node metastases: their prognostic relevance and relationship with head and neck squamous carcinoma primary sites. Clin Otolaryngol. 1994;19(1):63-9.

4. Merino OR, Lindberg RD, Fletcher GH. An analysis of distant metastases from squamous cell carcinoma of the upper respiratory and digestive tracts. Cancer. 1977;40(1):145-51.

5. Calhoun KH, Fulmer $P$, Weiss $R$, Hokanson JA. Distant metastases from head and neck squamous cell carcinomas. Laryngoscope. 1994;104(10):1199-205.

6. Al-Othman MO, Morris CG, Hinerman RW, Amdur RJ, Mendenhall WM. Distant metastases after definitive radiotherapy for squamous cell carcinoma of the head and neck. Head Neck. 2003;25(8):62933

7. Doweck I, Robbins KT, Vieira F. Analysis of risk factors predictive of distant failure after targeted chemoradiation for advanced head and neck cancer. Arch Otolaryngol Head Neck Surg 2001;127(11):1315-8

8. Vikram B, Strong EW, Shah JP, Spiro R. Failure in the neck following multimodality treatment for advanced head and neck cancer. Head Neck Surg. 1984;6(3):724-9.

9. Studer G, Seifert B, Glanzmann C. Prediction of distant metastasis in head neck cancer patients: implications for induction chemotherapy and pre-treatment staging? Strahlenther Onkol. 2008; 184(11):580-5.

10. Al-Othman MO, Morris CG, Hinerman RW, Amdur RJ, Mendenhall WM. Distant metastases after definitive radiotherapy for squamous cell carcinoma of the head and neck. Head Neck. 2003;25(8):629-33.

11. Ferlito A, Rinaldo A, Buckley JG, Mondin V. General considerations on distant metastases from head and neck cancer. ORL J Otorhinolaryngol Relat Spec. 2001;63(4):189-91.
12. Brouwer J, Bree R, Hoekstra OS, Langendijk JA, Castelijns JA, Leemans CR. Screening for distant metastases in patients with head and neck cancer: what is the current clinical practice? Clin Otolaryngol. 2005;30(5):438-43.

13. Johnson JT. Proposal of standardization on screening tests for detection of distant metastases from head and neck cancer. ORL J Otorhinolaryngol Relat Spec. 2001;63(4):256-8.

14. Brouwer J, de Bree R, Hoekstra OS, Golding RP, Langendijk JA, Castelijns JA, Leemans CR. Screening for distant metastases in patients with head and neck cancer: is chest computed tomography sufficient? Laryngoscope. 2005;115(10):1813-7.

15. Senft A, de Bree R, Hoekstra OS, Kuik DJ, Golding RP, Oyen WJ, Pruim J, van den Hoogen FJ, Roodenburg JL, Leemans CR. Screening for distant metastases in head and neck cancer patients by chest CT or whole body FDG-PET: a prospective multicenter trial. Radiother Oncol. 2008;87(2):221-9.

16. Younes RN, Gross JL, Silva JF, Fernandez JA, Kowalski LP. Surgical treatment of lung metastases of head and neck tumors. Am J Surg. 1997;174(5):499-502.

17. Buckley JG, Ferlito A, Shaha AR, Rinaldo A. The treatment of distant metastases in head and neck cancer-present and future. ORL J Otorhinolaryngol Relat Spec. 2001;63(4):259-64.

Recebido em 01/12/2008

Aceito para publicação em 02/02/2009

Conflito de interesse: nenhum

Fonte de financiamento: nenhuma

\section{Como citar este artigo:}

Dedivitis RA, Denardin OVP, Castro MAF. Fatores de risco para metástases a distância no câncer de cabeça e pescoço. Rev Col Bras Cir. [periódico na Internet] 2009; 36(6). Disponível em URL: http:// www.scielo.br/rcbc

\section{Endereço para correspondência:}

Rogério Aparecido Dedivitis

E-mail: dedivitis@hns@uol.com.br 